Mortéza Mahmoudian

Université de Lausanne

\title{
STRATIFICATION DES STRUCTURES LINGUISTIQUES
}

\author{
«Chaque siècle se moquait du précédent, l'accusant d'avoir généralisé \\ trop vite et trop naïvement. Descartes avait pitié des Ioniens ; Descartes à son \\ tour nous fait sourire; sans doute nos fils riront de nous quelque jour. \\ Mais alors ne pouvons-nous aller tout de suite jusqu'au but? N'est-ce \\ pas le moyen d'échapper à ces railleries que nous prévoyons ? [...] \\ Non, cela est impossible; ce serait méconnaître complètement le \\ véritable caractère de la science. »
}

H. Poincaré ${ }^{1}$

\section{Introduction}

Les thèses phonologiques du Cercle linguistique de Prague - une fois surmontées résistances et inerties - ont suscité un grand intérêt, et fait l'objet de nombreuses applications dans diverses langues. Elles aboutissent à l'élaboration de procédures simples et reproductibles qui permettent de dégager le matériel phonique des langues sans le confondre avec des faits purement physiques et qui correspond à l'intuition des sujets parlants. La phonologie acquiert ainsi le statut d'une science avec un objet et une méthode bien définis, et dont l'adéquation peut être appréciée par confrontation à des faits concrets.

Tout en éclairant l'essentiel de la structure et du fonctionnement phonologiques, ces procédures descriptives laissent des zones d'ombre, et soulèvent des questions. Deux problèmes sont au centre du présent exposé: $1^{\circ}$ structure et variations sont-elles antinomiques, $2^{\circ}$ quelles frontières délimitent une langue et la distinguent d'avec ses variétés ou usages?

\section{Originalité de la phonologie}

Les acquis de la phonologie et son statut de pionnière sont évidents. Je n'insisterai pas là-dessus, me bornant à signaler certaines caractéristiques qui en constituent — à mes yeux — l'apport théorique, et la distinguent des courants de pensée linguistique de l'époque.

La phonologie se donne pour objet l'étude des sons de la langue. Elle s'évertue à marquer la différence entre son en tant que fait de langue et son comme phénomène physique. Or, la confusion entre les deux objets était chose courante. ${ }^{2}$

Quant à la méthode, la phonologie part du principe que c'est la combinaison signifiant / signifié qui produit le signe linguistique; dès lors, toute modification du signifiant phonique entraîne une modification correspondante dans le signifié. Ce qui permet de concevoir l'épreuve de la commutation. ${ }^{3}$ Cette démarche

\footnotetext{
${ }^{1}$ PoINCARE (1918: 168).

2 Ainsi Daniel Jones (1950) qui définit le phonème comme une famille de sons, et récuse toute pertinence à l'opposition significative. Il maintient le refus de critères mentaux dans la réédition de 1967.

${ }^{3}$ Cette procédure a été initiée et pratiquée par Troubetzkoy bien avant que Hjelmslev ne lui applique le terme de commutation (HJELMSLEV, 1968).
} 
présente des avantages certains : $1^{\circ}$ elle est simple ou explicite, donc susceptible d'application, sans exégèse ni commentaire ; $2^{\circ}$ elle est reproductible, en ce sens que l'analyse du matériel phonique d'une langue quelconque peut être répétée aux fins de contrôle ; $3^{\circ}$ l'identité ou la différence des sons est établie sur le jugement intuitif du sujet parlant; ce qui rend la description indépendante des préjugés 'phonétistes' ${ }^{4}$ et $4^{\circ}$ le jugement d'identité ou différence doit émaner d'un individu parlant la langue à l'étude ; le descripteur peut être une personne autre, voire un linguiste qui n'est pas locuteur de cette langue. Soit dit en passant, ce dernier point a une importance capitale, car les Grecs anciens avaient accompli une analyse quasi phonologique de leur langue, comme en témoigne leur système orthographique. Mais leur analyse ne reposant pas sur des principes explicites, ils n'étaient pas en mesure d'appliquer les mêmes principes sur d'autres langues.

Cet appareil méthodologique a permis à la phonologie de procéder à des analyses avec certaine rigueur ${ }^{5}$, et d'obtenir une image du matériel phonique tel que le connaît et reconnaît le sujet parlant.

\section{Troubetzkoy et Saussure}

Pour caractériser pleinement la commutation, il convient d'ajouter qu'elle a aussi dans ses fondements une autre idée de base : les éléments constitutifs de la langue sont différentiels. On reconnaît dans cette démarche deux des principes chers à Saussure : le caractère oppositif (ou différentiel) des entités linguistiques et la combinaison signifiant/signifié, constitutive du signe linguistique. La commutation est née de la rencontre des deux principes.

En ce sens on peut dire, avec Jacqueline Fontaine (FONTAINE, 1974), que le Cercle linguistique de Prague est l'héritier de Saussure. Il convient de remarquer que tout en énonçant les principes fondateurs, Saussure ne procède pas à une synthèse qui débouche sur des procédures descriptives. C'est qu'il hésite entre deux conceptions de l'objet de la linguistique : selon la première, la langue est définie comme pure forme, indépendante de toute substance, ${ }^{6}$ alors que la seconde inclut dans la définition de la langue, des propriétés substantielles dont le caractère phonique du signifiant. ${ }^{7} \mathrm{Il} \mathrm{y}$ a là une contradiction dont résulte une bifurcation dans la voie tracée par Saussure. Hjelmslev, avec sa théorie glossématique, s'est résolument engagé dans la première voie, qui semble être une impasse comme en témoigne l'abandon où se trouve actuellement cette option théorique.

L'école de Prague a opté pour l'autre voie ; celle qui prend en compte et la forme et la substance pour identifier les éléments d'une langue. En cela, les Pragois n'ont pas suivi passivement l'enseignement de Saussure. Ils ont dû opérer un tri parmi les thèses saussuriennes, séparer la graine de l'ivraie. Au terme de

\footnotetext{
4 A l'époque, la théorie phonologique est mal reçue: elle est traitée, dans des rencontres internationales, avec mépris ou comme une curiosité : « tout le monde s'amuse énormément que dans une langue quelconque, deux sons aussi dissemblables soient perçus comme un seul et même phonème. » (TROUBETZKOY, 2006, lettre 130).

${ }_{6}^{5}$ Rigueur relative, certes. J’y reviendrai. Cf. infra $\$ 4$.

${ }^{6} \ll[\ldots]$ la langue est une forme non une substance[...] $»$ SAUSSURE (1916 : p. 169).

${ }^{7}$ Saussure considère que signifiant est « de nature auditive » donc linéaire (SAUSSURE, 1916: 103 \& 170).
} 
décennies d'expérience, ce choix s'est révélé judicieux; il a permis d'ouvrir des perspectives et de poser de nouveaux problèmes.

\section{Imprécisions et paradoxes}

Pour qualifier la méthode phonologique d'explicite (Cf. 2., supra), je me réfère à son cadre historique, celui des années 30 du siècle dernier. A cette époque, l'étude du matériel phonique de la langue a des bases subjectives; ainsi un Grammont affirme l'existence de 6 voyelles $a$ en français (GRAMMONT, 1935). Comment y arrive-t-il ? En se fiant à ses impressions auditives. La description phonologique suit une autre voie pour dégager les unités phoniques : on ne peut affirmer l'existence de deux phonèmes - disons /a/ et /â/ - que si l'observation met en évidence leur opposition dans des paires quasi homonymes comme las /lâ/ et la /la/, fasse /fâs/ et face /fas/, lasser /lâse/ et lacer /ase/, et ainsi de suite.

Les thèses pragoises sont explicites, comparées aux pratiques de l'époque; elles exigent cependant des développements et davantage d'explicitation à mesure qu'avancent les recherches phonologiques.

De quelle logique relèvent les unités phonologiques? Autrement dit, quel modèle est applicable en phonologie: le modèle déterministe ou le modèle stochastique (ou aléatoire)? Dans ses Principes de phonologie, Troubetzkoy récuse catégoriquement toute pertinence au nombre et à la mesure dans le domaine de la langue (TROUBETZKOY, $1949: 7-9),{ }^{8}$ excluant ainsi tout recours à la statistique. Ailleurs, dans le même ouvrage, il consacre des pages aux problèmes de statistique linguistique. A première vue, il s'agit d'un paradoxe.

Un accueil moins critique pourrait y trouver une cohérence : l'auteur aurait pu partir de l'idée que la phonologie d'une langue est un système complexe, constitué de sous-systèmes dont certains auraient une structure déterministe et d'autres une structure aléatoire. Dans le cas - improbable, me semble-t-il — où telle serait l'intention de Troubetzkoy, la théorie phonologique pècherait par manque d'explicitation suffisante.

A ces difficultés — imprécisions et paradoxes — s'en ajoute une autre ; celle des faits dont le statut ne peut être déterminé par la procédure qu'il propose pour l'analyse phonologique. Troubetzkoy part du principe - implicite - que l'opposition phonologique relève de la logique du oui ou non ${ }^{9}$ : deux sons s'opposent ou ne s'opposent pas. Sans moyen palier. Que faire alors des cas où l'on ne peut trancher la question de savoir si deux sons - /œ/ et /ö/, par ex. sont en opposition? C'est précisément le cas de deux linguistes français Georges Gougenheim (GougENHEIM, 1935) et André Martinet (MARTINET, 1933), acquis à la théorie phonologique et fondateurs de la Société française de phonologie - qui se trouvent en désaccord sur le système des voyelles françaises.

Le problème peut être formulé ainsi : la phonologie pragoise est fondée sur le principe que le système - phonologique, en l'occurrence — est de nature sociale, donc constant à travers la communauté linguistique. Les phénomènes variables ne relèvent donc pas du système. Or, l'épreuve de la commutation est censée révéler

\footnotetext{
${ }^{8}$ Troubetzkoy rejette le recours aux données statistiques dans sa polémique avec Zwirner. Qu'on ne me prête pas l'intention de soutenir la théorie phonométrique de celui-ci.

${ }^{9}$ D'où le rejet de la mesure et du nombre.
} 
les unités phonologiques. Dès lors, /œ/ et /ö/ ne sont pas des phonèmes, en raison de leur caractère variable. Or, en vertu de la commutation, les mêmes sons ont du moins pour une fraction des francophones - le statut de phonème.

Face à ce paradoxe, la phonologie pragoise reste démunie. L'enquête phonologique de Martinet (MARTINET, 1945) est une quête de réponse à ce genre de question. J'y reviendrai. Cf. infra $\$ 8$.

A ce point de l'exposé, un détour me semble utile pour signaler ou rappeler la façon dont science et conditions de scientificité sont conçues à l'époque.

\section{Structure et déterminisme}

L'idée même de structure ou de système est empruntée aux sciences 'dures'. Rappelons la comparaison que fait Karl Bühler entre les principes phonologiques de Troubetzkoy et le système du chimiste Mendeleïev (Cf. TROUBETZKOY, 2006, lettre 60, note 4). Ce qui fait de ce rapprochement un hommage appuyé, c'est le prestige des sciences de la nature, considérées à l'époque comme modèles à suivre. D'ailleurs, pour asseoir les bases d'une science du langage, les chercheurs de l'époque commencent leurs exposés par un plaidoyer pour le déterminisme, la causalité et contre le principe de libre arbitre. Ainsi Hjelmslev qui oppose au structuralisme la «tradition [humaniste] qui veut que les faits humains, contrairement aux faits de la nature, soient singuliers, individuels, et ne puissent donc ni être soumis comme ceux de la nature à des méthodes exactes, ni être généralisables. » (Hjelmslev, 1966, §2, p.17-18).

Rappelons que le déterminisme renvoie au «principe scientifique d'après lequel tout phénomène est régi par une (ou plusieurs) loi(s) nécessaire(s) telle(s) que les mêmes causes entraînent dans les mêmes conditions ou circonstances, les mêmes effets. » $(T L F i){ }^{10}$

La linguistique doit l'essentiel de ses acquis depuis le $\mathrm{XX}^{\mathrm{e}}$ siècle au concept de structure. L'emprunt a été bénéfique, malgré les réserves qu'on pourrait formuler sur certains des aspects ou certaines des applications du concept.

\section{Linguistique et sciences physiques}

Les sciences physiques ont été - j'aimerais insister là-dessus - source d'inspiration pour la linguistique dès le début du structuralisme. Le chapitre introductif du Langage de Leonard Bloomfield en est une bonne illustration. Bloomfield écrit : «Les actions humaines, selon la conception matérialiste, sont une partie des séquences de cause à effet, exactement comme celles que nous observons dans l'étude de la physique ou de la chimie. [...]. Nous pourrions prédire les actions d'une personne (si un certain stimulus l'amènera à parler et si oui, quels sont les mots exacts qu'elle prononcera, par exemple), à condition de connaître la structure exacte de son corps à ce moment, ou, ce qui revient au même, à condition de connaître la constitution exacte de son organisme lors d'un état antérieur — disons à la naissance ou avant — et que nous ayons ainsi un

\footnotetext{
${ }^{10}$ Voici la définition - sensiblement la même - qu'en donne Carl HOEFER : «The world is governed by (or is under the sway of) determinism if and only if, given a specified way things are at a time $t$, the way things go thereafter is fixed as a matter of natural law. »
} 
enregistrement de chaque changement de cet organisme, y compris de chaque stimulus qui ait jamais affecté cet organisme » (Bloomfield, $1970: 36$ )

Il est intéressant de comparer les positions de Bloomfield avec celles prises par Laplace, plus d'un siècle plus tôt : «Une intelligence qui, pour un instant donné, connaîtrait toutes les forces dont la nature et la situation des êtres qui la composent est animée, si d'ailleurs elle était assez vaste pour soumettre ces données à l'analyse, embrasserait dans la même formule les mouvements des plus grands corps de l'univers et ceux des plus légers atomes : rien ne serait incertain pour elle, et l'avenir comme le passé serait présent à ses yeux. L'esprit humain offre, dans la perfection qu'elle a su donner à l'astronomie, une faible esquisse de cette intelligence. » (LAPLACE, 1814)

La similitude est frappante entre Laplace - à qui remonte l'idée de déterminisme - et Bloomfield, même si celui-ci ne parle pas de déterminisme, mais de «théorie matérialiste (ou mieux mécaniste) [...]» BLOOMFIELD (1970:36)

\section{Déterminisme absolu}

Dans la perspective philosophique, le concept de déterminisme pose de nombreux problèmes. Des débats nourris mettent à jour de multiples acceptions du terme, dont je relève deux qui ont trait à notre thème : $1^{\circ}$ le déterminisme absolu «défini par la prévisibilité rigoureuse» et $2^{\circ}$ «la régularité globale, macroscopique, résultant d'une loi de probabilité à laquelle sont soumis un grand nombre d'événements indépendants, c'est le déterminisme statistique » (BALIBAR \& MACHEREY, 2009).

Dans la littérature des sciences physiques, le terme de déterminisme renvoie - depuis le $\mathrm{XIX}^{\mathrm{e}}$ siècle jusqu'à nos jours — au déterminisme absolu excluant ainsi l'aléatoire (LESNE, 2008). Les phénomènes de la nature sont ainsi censés être régis par des lois déterministes, applicables à tout un domaine d'objets sans exception aucune. Il s'ensuit qu' «un seul contrexemple, dans un domaine précis de faits, suffit à ruiner une hypothèse générale s'il est fermement assuré et si vraiment il prend l'hypothèse en flagrant délit de prédiction fausse. Telle est l'une des grandes vertus de la méthode scientifique. Dans le passé, même des constructions admirables [...] se sont assez souvent effondrées ainsi sous l'effet d'une réfutation par des faits très particuliers » (ESPAGNAT, 1981:4). C'est dire que dans les sciences physiques, jusqu'au début du $\mathrm{XX}^{\mathrm{e}}$ siècle, la structure est déterministe — dans l'acception stricte du terme — ou n'est pas.

On comprend dès lors que la structure, concept emprunté aux sciences 'dures', soit conçue conforme au déterminisme absolu.

\section{Variations structurées}

Revenons au paradoxe de la phonologie. Cf. supra §3. Pour s'en sortir, on peut envisager diverses solutions. Une solution - radicale - serait de remettre en cause les fondements théoriques et les acquis de la phonologie. Ce qui semble correspondre à l'attitude de certains linguistes du courant transformationnaliste. Mais faut-il jeter l'enfant avec l'eau de bains ? 
Une autre consisterait à accréditer d'une valeur absolue, la méthode, et de reléguer au rang de la parole (distincte de la langue), de l'usage (opposé à la structure) ou de la performance (par opposition à la compétence) tous les faits observables qui ne cadrent pas avec le modèle pré-établi. Dans ce cas, la cohérence de la description sera sauve. Mais ce faisant, ne sacrifie-t-on pas l'adéquation empirique de la théorie?

Il y a encore une autre solution, celle de parier sur le dialogue expérimental : la théorie permet de prévoir le comportement de l'objet. Quand les phénomènes observés ne correspondent pas aux prévisions, de deux choses l'une : ou bien les hypothèses ne sont pas adéquates à l'objet (ou du moins, à l'ensemble d'un domaine d'objets) ou bien les faits sont contestables, en ce qu'ils ne sont pas observés dans des conditions valables.

L'enquête phonologique permet d'apprécier la valeur de l'hypothèse sur la foi de ses applications. Quand les sujets d'une langue, interrogés dans des conditions identiques, émettent des jugements variés sur l'identité ou la différence phonologiques, il convient de remettre en cause l'hypothèse. En l'occurrence, l'hypothèse de l'invariabilité des phénomènes phonologiques à travers la communauté parlante.

\section{Résultats d'enquête}

L'enquête phonologique de Martinet (MARTINET, 1945) apporte des éléments permettant de pondérer le rapport entre constance et variations dans une langue. Plusieurs constats s'imposent :

- la variation touche toutes les unités soumises à l'enquête ;

- l'ampleur des différences varie suivant les facteurs géographiques $;^{11}$

- le nombre et les caractéristiques des variétés d'usage dépendent de la finesse de l'analyse ;

- à la limite, on peut établir un système phonologique distinct pour chaque informateur si on prend en compte toutes les différences, même infimes.

Ces résultats ébranlent l'idée d'un système linguistique constant à travers toute la communauté. Tenant compte de ces données, on ne peut plus soutenir l'idée que «La langue existe dans la communauté sous la forme d'une somme d'empreintes déposées dans chaque cerveau, à peu près comme un dictionnaire dont tous les exemplaires, identiques, seraient répartis entre les individus » (SAUSSURE, 1916, p. 38).

Les résultats d'enquêtes nous invitent à concevoir la langue non comme une structure homogène, mais bien comme une structure comportant des variations inhérentes; autrement dit une structure stratifiée où les strates sont hiérarchisées suivant leur plus ou moins grande extension dans la communauté. Ce qui a pour implication, entre autres, que langue et variété ne pourraient être séparées par des limites nettes.

D'où des questions sur les rapports entre structure et fonction ainsi que sur la délimitation de la langue.

\footnotetext{
${ }^{11}$ La population enquêtée étant relativement homogène du point de vue de social, les résultats ne reflètent pas les variations potentielles dues aux facteurs sociaux.
} 


\section{Structure et fonction}

On conçoit généralement la langue comme un instrument de communication. Il s'ensuit que pour assurer sa fonction, elle doit être stable, invariable à travers la communauté. Comment une langue peut-elle incorporer des variations sociales et servir à communiquer? N'y a-t-il pas contradiction entre les deux propriétés attribuées à la langue. Autrement dit, admettre la variabilité de la langue n'implique-t-il pas l'incommunicabilité ?

Certes. Mais l'objection ne serait valable que si la communication était une fonction absolue; c'est-à-dire si la communication était soit possible soit impossible. Or, il y a des degrés d'intercompréhension. Un Parisien comprend bien un autre Parisien ; comprend-t-il avec une égale facilité un Marseillais, un Québécois, ... ? Quand on se déplace à travers un espace linguistique, on se heurte parfois à des difficultés d'intercompréhension; difficultés croissantes à mesure qu' augmente la distance - géographique ou sociale - entre les interlocuteurs. A quoi sont dues ces difficultés?

Il est raisonnable de voir dans les variations structurales l'une des causes de l'intercompréhension graduelle. On pourrait émettre l'hypothèse que plus les écarts entre usages en contact sont grands et plus les difficultés d'intercompréhension sont importantes. La vérification d'une telle hypothèse est en soi une recherche conséquente.

Non seulement la structure stratifiée n'est pas incompatible avec la fonction communicative, elle peut contribuer à en expliquer le processus, et à en mesurer les degrés. L'adaptation pourrait en fournir une illustration : si un Vaudois et un Marseillais se comprennent, c'est parce qu'ils font varier leur usage selon les circonstances. C'est dire que tout sujet parlant est conscient des variétés de sa langue, et qu'il est en mesure d'adapter peu ou prou la sienne à celle de son interlocuteur.

\section{Et pourtant, elle existe}

Que devient la langue dans tout cela ? Elle est à la fois insaisissable et indispensable.

Insaisissable, car ce qu' on appelle une langue - le français, par ex. - est un conglomérat d'usages: parisien, toulousain, valaisan,... Indispensable, parce qu'en passant du français à l'allemand, le sujet parlant a l'impression de faire quelque chose de fondamentalement différent de l'adaptation de sa langue aux circonstances.

Le problème provient du décalage entre fait d'expérience et résultat de procédures descriptives. La langue existe en vertu de l'observation de l'intuition et du comportement du sujet parlant. La langue n'existe pas si l'on se fie au résultat de l'application conséquente d'une méthode.

Le problème est de savoir si le résultat de la recherche scientifique est le réel ou s'il ne fait que représenter un ou plusieurs des aspects du réel. C'est un problème philosophique - vieux, mais toujours actuel - qui dépasse le cadre du présent exposé. Je ne l'aborderai pas, me bornant à soulever un problème. Actuellement, les méthodes scientifiques en biologie ne semblent susceptibles de proposer aucun moyen pour tracer une frontière claire entre le vivant et la matière 
inerte. Faut-il en conclure que le vivant et le non vivant ne constituent pas deux catégories distinctes?

Peu importe que les deux extrêmes - langue et idiolecte - ne soient pas séparés par des frontières nettes, les deux objets restent distincts. De même que pour l'exercice de ses activités, le paysan distingue le marécage, le pierrier et la surface labourable, bien que l'humidité du sol et la teneur en cailloux varient graduellement. De même, au niveau de la pratique quotidienne, la distinction avec une approximation tolérable - entre langue et variété est possible. Si la linguistique vise à décrire et à expliquer la façon dont l'usager connaît et reconnaît les outils linguistiques dont il se sert, elle doit rechercher les procédures d'approximation auxquelles il a recours.

\section{Comment distinguer langue et usage ?}

Si un Lillois et un Niçois reconnaissent comme français l'usage l'un de l'autre - malgré les différences qui ne leur échappent pas —, c'est qu'il existe des caractéristiques structurales qui leur servent de critères. Quelles sont ces caractéristiques ?

C'est un problème qu'on doit traiter avec sérieux. Une idée assez répandue est que les usages auraient une structure aléatoire par opposition aux langues qui, elles, seraient dotées d'une structure déterministe. En d'autres termes, les usages seraient sujets à des fluctuations imprévisibles, alors que les langues ne souffriraient aucune variation structurale. Il n'en est rien; et on peut constater «que tous les membres d'une telle communauté [linguistique] ne parlent pas de façon identique et que les divergences peuvent s'étendre à certains points de la structure de la langue. » (MARTINET, 1960 : § 5.1.)

Probablement, des travaux - empiriques et de réflexion - seront nécessaires pour identifier la frontière qui les sépare. On pourrait risquer une hypothèse : puisque langue et usage sont toutes deux susceptibles de variations, leur distinction serait fondée sur l'amplitude de leurs variations respectives. Autrement dit, il y aurait un niveau macroscopique, celui des langues, caractérisé par une faible indétermination alors que les usages - situés au niveau microscopique se distingueraient par une forte indétermination.

C'est sans doute cette faible indétermination qui fait que la structure déterministe sans valoir strictement pour les langues, est cependant un modèle avec une approximation raisonnable; et c'est le faible écart entre le modèle déterministe et la structure linguistique qui a permis à la phonologie de réaliser des analyses et descriptions suffisamment précises pour être convaincantes, et d'assurer des avancées non négligeables.

\section{Implications des enquêtes}

Les enquêtes phonologiques - de Martinet (MARTINET, 1945) et de Labov (LABOV, 1976), par exemple - apportent des arguments en faveur d'une révision du concept de structure linguistique.

Considérons en survol les techniques descriptives : à l'origine, les oppositions phonologiques étaient conçues comme des évidences, ainsi qu'en témoignent les écrits de Troubetzkoy ou de Bloomfield. Le souci d'élaborer précisément les 
techniques a amené des linguistes à prôner le recours à l'intuition du sujet parlant ; mais ce recours est - pour certains dont Harris (HARRIS, 1951) - limité à un jugement sur l'équivalence ou la différence entre deux séquences phoniques : xyz $=\mathrm{xwz}$ ? Ou bien xyz $\neq \mathrm{xwz}$ ? Constatant les variations à l'intérieur d'un 'même' système phonologique, Martinet a recours à l'enquête. Il n'est pas sans intérêt de remarquer que son questionnaire ne limite pas le rôle de l'informateur au jugement «identique ou différent?». Par exemple, le sujet est sollicité de juger de certaines qualités phoniques des oppositions : longueur vocalique ou timbre par ex. Il y a, sous-jacentes à ces questions, plusieurs hypothèses dont : $1^{\circ}$ le savoir linguistique du sujet parlant dépasse de beaucoup le seul fait d'identité ou de différence $; 2^{\circ}$ il a conscience de certaines propriétés physiques des phonèmes ${ }^{12} ; 3^{\circ}$ il sait que sa pratique linguistique a des variétés ${ }^{13}$ et $4^{\circ}$ il est à même d'appeler cette connaissance à la conscience, et de l'exprimer.

Labov pousse encore plus loin la finesse de l'outil descriptif au moins sous deux aspects : confronter la pratique linguistique déclarée et la pratique observée, d'une part et de l'autre, repérer les reflets des clivages sociaux d'une langue dans le jugement intuitif de l'usager.

Les nouvelles techniques produisent deux effets opposés. Elles permettent de décrire de façon de plus en plus adéquate, la pratique linguistique des fractions de plus en plus restreintes; et en même temps, elles creusent l'écart entre la description qu'elles fournissent et la pratique linguistique d'autres fractions. Dès lors, elles ne sont pas adéquates à la description de ce qui est commun aux usages de la communauté globale, et qu'on appelle la langue.

La diversité des techniques a — à mon avis — des implications théoriques dont la moindre n'est pas que les procédures descriptives doivent être appréciées non dans l'absolu, mais relativement à l'objet étudié et le but visé.

\section{Convergences et divergences}

L'identification, dans les sciences physiques, de la structure avec le déterminisme n'est remise en cause que vers les années 1920-30 avec la métamorphose de la physique et l'avènement de la mécanique quantique.

L'évolution de la linguistique et celle de la physique font apparaître un parallélisme sous au moins deux aspects : la fragmentation de l'objet d'étude et la complexité de sa structure. En physique, on passe de l'atome (censément indivis) à l'univers subatomique des particules élémentaires, des baryons, des quarks, des préons, etc. Et en linguistique, on passe de la langue (censée être partagée par toute une communauté) à des variétés (ayant des aires de plus en plus restreintes) jusqu'aux idiolectes en passant par sociolectes, ethnolecte, basilectes, mesolectes, acrolectes,...

Au niveau de la description aussi on peut observer une évolution parallèle : on passe des structures simples à des structures de plus en plus complexes; ce qui implique qu'on révise les méthodes d'analyse, et qu'on en introduise de nouvelles.

\footnotetext{
${ }^{12}$ Cf. Martinet (1945), la question 8, p. 11, qui porte sur la nature physique d'une opposition : timbre ou longueur.

${ }^{13}$ Cf. MARTINEt (1945), la question 3, p. 11 par laquelle il est demandé à l'informateur de fonder ses réponses sur « le parler tout à fait naturel et familier».
} 
Le parallélisme irait-il plus loin sans que la linguistique risque de singer la physique? Je ne crois pas qu'on puisse y répondre sans circonspection, l'interprétation et l'application du concept de déterminisme variant beaucoup en passant de la physique à la linguistique.

En physique, le modèle est strictement élaboré, et rigoureusement contrôlé par l'expérimentation. Tel n'est pas le cas en linguistique. Saussure et Bloomfield, par exemple, qui déploient des efforts pour fonder une «science du langage », ne parviennent pas à définir des concepts univoques permettant un dialogue expérimental concluant. Par souci d'objectivité, — peut-être aussi pour éviter les pièges prescriptifs - ils définissent la langue en termes de la communauté linguistique, et la conçoivent comme un système fermé. Or, en appliquant ces principes, ils arrivent au constat que les frontières d'une communauté linguistique ne sont pas nettes. Dès lors la langue a aussi des limites floues; elle n'est donc pas un système clos. ${ }^{14}$ L'absence de clôture constitue un inconvénient majeur : le chercheur n'est jamais sûr que les cas qu'il considère sont dans la structure à l'étude et non en dehors d'elle. Dès lors, la construction de modèles déterministes avec des hypothèses réfutables n'est qu'un voeu pieux, dépourvu de toute perspective opératoire.

Si l'on cherche véritablement le dialogue expérimental, mieux vaut partir de modèles aléatoires ; on aura des chances d'apprécier les hypothèses concurrentes, et d'adopter celle qui se révèle la plus adéquate à l'objet observé.

La prise en compte des variétés implique une rupture méthodologique. Ainsi sont remis en cause les principes fondateurs, à commencer par l'homogénéité et la clôture.

Exemple : le phonème est défini, dans le modèle classique, par sa fonction distinctive, donc par ses oppositions mises en évidence par l'épreuve de la commutation. Or, dans les situations de contact, une telle procédure est inopérante; il faut donc établir de nouvelles procédures. Ainsi, l'étude de Weinreich (WEINREICH, 1953) met en évidence l'importance de la substance phonique, seul repère tangible et immédiat. C'est de là que l'apprenti locuteur peut partir pour construire une phonologie pour sa nouvelle langue - identifier les unités, reconnaître les oppositions - par tentatives et erreurs, et avec plus ou moins de bonheur, selon les cas.

Pour expliquer les processus linguistiques complexes sur ce terrain mouvant, semé d'embûches, le modèle classique d'analyse phonologique n'est guère adéquat. Il doit être réexaminé au moins sous trois aspects :

a/ modifications de modèles descriptifs. La réintégration des éléments variables entraîne des modifications de modèles descriptifs ;

$b /$ pluralité des modèles descriptifs. Dès lors, ce sur quoi la linguistique doit se pencher n'est pas un objet et une méthode uniques, mais bien une famille d'objets et un ensemble de modèles descriptifs ; lesquels modèles sont plus ou moins applicables aux divers membres de la famille ; et

c/ complémentarité. L'introduction de nouveaux modèles descriptifs n'abolit pas le modèle classique. Ils sont complémentaires, chacun ayant son domaine

\footnotetext{
${ }^{14}$ Cf. Mahmoudian (1980) et Mahmoudian (1990).
} 
d'application propre. ${ }^{15}$ De cette complémentarité découle que les linguistes devront - suivant l'échelle et le but de l'analyse - utiliser l'un ou l'autre modèles.

\section{BIBLIOGRAPHIE}

BALIBAR Etienne \& MACHEREY Pierre (2009), Déterminisme, Encyclopaedia Universalis.

BLOOMFIELD Leonard (1970), Le langage, Paris, Payot, (édition anglaise 1935).

Bourgine Paul, Chavalarias David, COHEN-Boulakia Claude (2008),

Déterminismes et complexités : du physique à l'éthique. Autour d'Henri Atlan,

Paris, Ed. La Découverte.

ESPAGNAT Bernard de (1981), A la recherche du réel, Paris, Gauthier-Villars.

FONTAINE Jacqueline (1974), Le cercle linguistique de Prague, Paris, Mame.

GougENHEIM Georges (1935), Eléments de phonologie française, ParisStrasbourg, Belles Lettres \& Publications de la Faculté des lettres de l'Université de Strasbourg.

GRAMMONT Maurice (1935), Traité pratique de prononciation française, Paris, Delagrave (première édition 1916, réédité en 1972).

HARRIS Zellig S. (1951), Structural Linguistics, Chicago, Chicago University Press.

HJelmSleV Louis (1968), Prolégomène à une théorie du langage, Paris, Minuit (éd. origin. 1943).

HoEFER Carl, Detrminism, Stanford Encyclpaedia of Philosophy, I. Introduction. En ligne : [http://plato.stanford.edu/entries/determinism-causal/]

JONES Daniel (1950), The Phoneme: Its Nature and Use, Cambridge, Heffer (réédité en 1967).

LABOV William(1976), Sociolinguistique, Paris, Minuit.

LAPLACE Pierre-Simon de (1814), Essai philosophique sur les probabilité, Courcier, Paris.

LESNE Annick (2008), Déterminisme et aléatoire dans les systèmes complexes : un faux débat? In : Bourgine, CHAVALARIAS, COHEN-BOULAKIA (2008).

MAHMOUdian Mortéza (1980), Structure linguistique : Problèmes de la constance et des variations, in : La Linguistique, vol. 16, fasc. 1, p. 5-36.

MAHMOUDIAN Mortéza (1990), Linguistique et sociolinguistique, La Linguistique, vol. 26, Fasc. 2, p. 47-76.

MARTINET André (1933), Remarques sur le système phonologique français, BSL, 34, p. 191-202.

MARTINET André (1945), La prononciation du français contemporain, Genève, Droz.

MARTINET André (1960), Eléments de linguistique générale, Paris, Armand Colin. POINCARÉ Henri (1918), Science et hypothèse, Paris, Flammarion.

\footnotetext{
${ }^{15}$ Cette complémentarité a pour conséquence d'éviter deux positions extrêmes : celle qui ne retient comme pertinents que les éléments constants et aussi celle qui privilégie les éléments variables. Cf. MAHMOUDiAn (1980 : 74).
} 
Trésor de la langue française (en ligne : TLFi), [http://atilf.atilf.fr/tlf.htm]. SAUSSURE Ferdinand de (1916), Cours de linguistique générale, Paris, Payot. TroubetZKoy N. S. (1949), Principes de phonologie, Paris, Klincksieck.

TroubetZKoy N. S. (2006), Correspondance avec Roman Jakobson et autres écrits, édition établie par Patrick Sériot, Lausanne, Editions Payot.

WeINREICH Uriel (1953), Languages in Contact, Publications of the Linguistic Circle of New York, $n^{\circ} 1$.

\section{SUMMARY}

The Praguean phonology is the first to have elaborated a repeatable procedure to describe the sound pattern of languages. This procedure allows to single, with some rigor, speech sound in contradistinction with sound as physical phenomenon. However, this procedure leaves a few problems unresolved. This paper aims at discussing two of these: $1^{\circ}$ the antinomy of deterministic structure and random (i.e. stochastic) structure and its implications for the function of language as communication tool; $2^{\circ}$ the frontiers between a language as such and its dialects. 